\title{
Tratamiento teórico-experimental de la paráfrasis: análisis de su uso con y sin el marcador discursivo o sea en español
}

\author{
Shima Salameh JimÉnez \\ Profesora Asociada \\ Universidad Jaime I de Castellón \\ Grupo Val.Es.Co \\ Facultad de Humanidades y Ciencias Sociales \\ Av. De Vicent Sos Baynat s/n \\ 12071 Castellón de la Plana \\ salameh@uji.es
}

TRATAMIENTO TEÓRICO-EXPERI-
MENTAL DE LA PARÁFRASIS: ANÁLI-
SIS DE SU USO CON Y SIN EL MARCASIS DE SU USO CON Y SIN EL MARCA-
DOR DISCURSIVO O SEA EN ESPAÑOL

RESUMEN: Este trabajo describe la relación de paráfrasis desde un enfoque teórico-experimental. Parte de la idea de que la paráfrasis (la equivalencia) (E. Gülich y Th. Kotschi, 1983, 1995) no es una actividad de fácil producción en comparación con la reformulación discursiva, una postura distinta a la defendida en los estudios publicados durante los últimos cuarenta años. Para completar esta visión, se aplica la técnica experimental de lectura con eye-tracking, que permite medir cuántos esfuerzos cognitivos requiere un proceso de asimilación informativa a partir de los movimientos oculares producidos (hipótesis ojo-mente, K. Rayner, 1998). Los datos obtenidos complementan el tratamiento de funciones discursivas como paráfrasis, reformulación o corrección, entre otras, cuyos limites definitorios no quedan claros (S. Pons, 2013).

PALABRAS CLAVE: paráfrasis; reformulación; experimental; eye-tracking; marcadores discursivos.

SUMARIO: 1 . Introducción. 2 . Paráfrasis e igualdad. Algunas nociones básicas. 2.1. Definición, características y problemas. 2.2. Eye-tracking y pragmática experimental. 2.3. Hipótesis teórico-experimentales. 3. Metodología. 3.1. Movimientos oculares y duración. 3.2. Diseño del experimento. 4. Patrón cognitivo de la relación de equivalencia en paráfrasis. Datos. 5 . Conclusiones.

\section{THEORETICAL-EXPERIMENTAL APPROACH TO PARAPHRASE WITH/WITHOUT THE REFORMU- LATION MARKER OSEAIN SPANISH}

ABSTRACT: This study explores paraphrastic reformulation from a theoretical-experimental approach: it is defended that paraphrase is not a discourse activity to be easily produced and processed. Previous approaches defend the contrary approach: reformulation is a harder function since it is not based on equivalence but distance between formulations. This paper presents quantitative and qualitative results showing that paraphrase involves hard processes despite being based on an equivalence between two formulations (E. Gülich \& Th. Kotschi, 1983 1995). Eye-tracking experimental techniques allow measuring cognitive efforts behind information assimilation by addressing ocular movements and their duration (eye-mind hypothesis, K. Rayner, 1998). Data obtained will complement theoretical proposals accounting for paraphrase, reformulation or correction, which are unclearly defined (S. Pons, 2013).

KEYWORDS: paraphrase; reformulation; experimental; eye-tracking; discourse markers.

SUMMARY: 1. Introduction. 2 . Paraphrase and equivalence. Theoretical framework. 2.1. Definition, features and issues. 2.2. Eye-tracking and Experimental Pragmatics. 2.3. Theoretical-experimental hypotheses. 3. Methods. 3.1. Ocular movements and duration. 3.2. Experimental design. 4. Paraphrase and equivalence cognitive pattern. Data. 5. Conclusions.

\author{
APPROCHE THÉORIQUE-EXPÉ- \\ RIMENTALE DE LA PARAPHRASE \\ AVEC/SANS LE MARQUEUR DE RE- \\ FORMULATION O SEA EN ESPAGNOL
}

RÉSUMÉ: Cette étude explore la reformulation paraphrastique d'une visée expérimentale à travers des méthodes de eye-tracking. Le eye-tracking permet de mesurer les efforts cognitifs derrière l'assimilation de l'information à partir de l'étude des mouvements oculaires et de leur durée (hypothèse peil-esprit. K. Rayner, 1998). Contrairement à la littérature précédente, les résultats qualitatifs de cet étude montrent que la paraphrase, même si sert à indiquer une équivalence entre deux formulations (E. Gulich \& Th. Kotschi, 1983, 1995), n'est pas une activité discursive simple. Les données sur la paraphrase présentées ici s'ajoutent à des autres études sur des categories voisines (voire la reformulation ou la correction), qui ne sont pas non plus clairement définies (piège forme-fonction, S. Pons, 2013).

MOTS-CLÉS: paraphrase ; reformulation ; experimental ; eyetracking ; marqueurs du discours.

SOMMAIRE: 1. Introduction. 2. Paraphrase et équivalence. Cadre théorique. 2.1. Définition, caractéristiques et problèmes. 2.2. Eye-tracking et pragmatique expérimentale. 3. Méthodologie. 3.1. Mouvements oculaires et durée. 3.2. Préparation de l'experiment. 4. Paraphrase et modèle cognitive de l'équivalence. 5 . Conclusions. $\begin{array}{ll}\text { Fecha de Recepción: } & 30 / 11 / 2020 \\ \text { Fecha de Revisión: } & 19 / 01 / 2021 \\ \text { Fecha de Aceptación: } & 14 / 07 / 2021 \\ \text { Fecha de Publicación: } & 01 / 12 / 2021\end{array}$ 
Tratamiento teórico-experimental de la paráfrasis: análisis de su uso con y sin el marcador discursivo o sea en español ${ }^{1}$

Shima Salameh JimÉneZ

\section{INTRODUCCIÓN}

En las últimas décadas, la reformulación y otras funciones cercanas (paráfrasis, conclusión, corrección, resumen, ampliación, reducción, etc.) se han convertido en objeto de estudio de la pragmática teórica. Algunos de los temas abordados en este campo han sido la relación entre estas funciones y los marcadores discursivos que las expresan (E. Roulet, 1987; C. Rossari, 1994), el peso de la semántica o la polifonía en su producción (C. Noren, 1999; S. Murillo, 2007, 2016), su estatus como relaciones discursivas (C. Fuchs 1994) y, especialmente, su descripción pragmática general (E. Gülich y Th. Kotschi, 1983a, b, 1995; E. Roulet, 1987) en numerosos trabajos que, finalmente, se han planteado cuáles son sus limites definitorios (S. Pons, 2013, 2017).

Sobre su caracterización, otro de los aspectos tratados es el de la complejidad/no complejidad subyacente a las nociones de igualdad y distancia y su establecimiento. Concretamente, concebir la paráfrasis (o reformulación parafrástica) desde un tratamiento formal implica una visión estricta de la igualdad, aplicable solo a enunciados cuyas formas lógicas y condiciones de verdad sean idénticas (Smaby, 1971: 2). En este sentido, su producción sería sencilla: si uno de estos dos parámetros semánticos no se cumple, no existe paráfrasis. Sin embargo, la aplicación de esta visión a los usos reales de paráfrasis entre formulaciones refleja una relación más compleja: los contextos tratados desde una perspectiva pragmático-discursiva muestran una gran variedad de casos marcados por el contexto que no encajan en una dicotomía paráfrasis/no paráfrasis tan cerrada.

Para que se produzca una paráfrasis, los participantes en una interacción deben detectar (i) que dos contenidos se proponen como iguales a partir de unos pocos rasgos similares (predicación de identidad, M. F. Mortureux, 1982: 52), y (ii) que dicha equivalencia se establece gracias a una intención comunicativa, puesto que no tiene sentido parafrasear si no existe una necesidad de aclarar el significado de algo que ya se ha dicho. Por tanto, la noción de igualdad es más compleja que una simple relación de equivalencia entre contenidos, y en contextos menos claros la sitúa incluso cerca de la reformulación.

Todo esto conduce a la idea de que existe un conjunto de procesos discursivos y cognitivos, de producción y asimilación, que deberian demostrar que las relaciones de equivalencia semántico-pragmática presentan un grado de dificultad mayor al defendido en la bibliografia. Actualmente, la pragmática experimental ofrece herramientas que

1 Este artículo ha sido posible gracias al proyecto de investigación UDEMADIS, Unidades discursivas para una descripción sistemática de los marcadores discursivos en español (FFI-2016-77841-P), financiado por el Ministerio de Economía y Competitividad, AEI y los Fondos FEDER, y por la red de investigación REDT (FFI-2017-90738 REDT), financiada también por el Ministerio de Economía y Competitividad y AEI. 
permiten testar razonamientos teóricos de estas características. Por ello, en este trabajo aplicamos la técnica experimental eye-tracking $(\mathrm{M}$. A. Just y P. A. Carpenter 1980: 336), ya empleada en trabajos similares con buenos resultados (A. López Serena y Ó. Loureda, 2013: 221). Esta técnica parte de la hipótesis ojo-mente, por la que existe una relación directa entre la cantidad y el tipo de movimientos oculares producidos al observar un contenido determinado (imágenes, textos, párrafos, enunciados o palabras) y su asimilación (K. Rayner, 1978: 621).

Mediante la aplicación de esta técnica se abordan dos objetivos: el primero, analizar diferentes contextos parafrásticos, expresados con y sin marcador de reformulación, para comprobar si la relación de igualdad implica o no costes de procesamiento altos. La presencia de costes elevados indicaria que, efectivamente, la noción de igualdad es más compleja de lo que pudiera parecer. El segundo, medir cuál es el peso de los marcadores de reformulación en el establecimiento de la igualdad y si, en línea con algunos trabajos teóricos, su presencia facilita o no la producción-asimilación de esta función (C. Rossari 1990: 345). Este trabajo pretende aportar una nueva dimensión analitica y experimental para algunas de las ideas ya exploradas en el campo de la reformulación en español que, sin embargo, todavía dejan algunas cuestiones abiertas.

\section{Paráfrasis e igualdad: Algunas nOCIONES bÁSicas}

Aunque en las siguientes subsecciones abordamos nuestro objeto de estudio a partir de las denominaciones "paráfrasis" e "igualdad", la más habitual en este campo ha sido "reformulación parafrástica" (E. Gülich \& Th. Kotschi 1983). Evitamos esta etiqueta con el fin de no caer en la trampa forma-función (Pons 2013, 2017), estudiada en trabajos recientes para el español (S. Pons Bordería 2013) o el francés (H. Vassiliadou 2019), y según la cual la reformulación no debe confundirse con otras categorias cercanas, como la paráfrasis, la conclusión o la corrección, únicamente por el hecho de expresarse con los mismos marcadores, o por una tendencia de denominación en las publicaciones (H. Vassiliadou 2019: 12) por oposición a "reformulación no parafrástica" (E. Roulet 1987). De este modo, no asociamos la intención comunicativa propia de una paráfrasis, que es la de aclarar o mejorar la comprensión de un contenido concreto, con la de una reformulación, que es marcar una distancia clara con respecto a un contenido previo en un grado tan alto que imposibilita una identificación entre formulaciones.

Es importante que ambas intenciones comunicativas queden bien delimitadas para no añadir más dificultad de caracterización a las nociones "paráfrasis" e "igualdad". Si el lector analizara los ejemplos y datos presentados como posibles tipos de reformulación, podría ver en ellos una base de distancia (E. Roulet 1986: 195; E. Roulet 1987: 117) que, si bien puede ser mínima en el terreno de la enunciación, no existe desde un punto de vista semántico-pragmático, o no, al menos, en un grado completo, como se verá. 


\subsection{Definición, CaRacterísticas y PROBlemas}

La paráfrasis suele definirse como "una relación semántica" (E. Gülich y Th. Kotschi 1987: 30), aunque también pragmática (C. Fuchs 1994: 2), y, precisamente por esto, se concibe como "estrategia comunicativa resultante de una mala formulación de una idea o contenido" (E. Gülich y Th. Kotschi 1983: 341) que pretende "resolver problemas comunicativos de comprensión, problemas con la figura del interlocutor e incluso de amenaza de imagen" (1983: 305-308; M. Murat y B. Cartier-Bresson 1987: 6) en discursos conversacionales y textuales (M. Charolles y D. Coltier 1986: 51). Estas definiciones comparten un rasgo: describen la paráfrasis como una operación que "surge de un proceso de auto-reflexión sobre la lengua" (C. Bach 2000: 71) y que busca facilitar la progresión discursiva y paliar posibles defectos comunicativos de cualquier discurso (G. Antos 1982).

Sin embargo, es llamativo que, a pesar del acuerdo existente a la hora de tratarla como operación semántico-pragmática (de la que puede desprenderse un cierto grado de dificultad), estos mismos trabajos reflejen una visión de la paráfrasis aparentemente sencilla en comparación con la reformulación no parafrástica. Un meta-análisis de algunas referencias publicadas en este campo apoya esta idea:

(i) Las primeras definiciones de reformulación no parafrástica parten de dos conceptos esenciales: completitud interactiva (E. Roulet 1986) y discurso como negociación (E. Roulet 1987). Básicamente, una reformulación se produce cuando los hablantes tienen una necesidad de distanciarse de sus propias formulaciones para negociar el sentido del discurso y poder comunicarse exitosamente con sus interlocutores. Por lo general, estos dos conceptos no están relacionados con la paráfrasis.

(ii) En estos mismos trabajos, se define la reformulación no parafrástica con base en diversos niveles jerárquicos del discurso. La presencia de estos niveles más complejos se debe a la intención de negociar y completar el discurso (Roulet 1986) que, a priori, no se aplica a la noción de equivalencia, caracterizada estructuralmente como la vinculación de "dos constituyentes en un mismo nivel jerárquico", y que como "una simple paráfrasis" (E. Roulet 1987: 115).

(iii) Los conceptos de completitud y negociación no se relacionan habitualmente con la paráfrasis, ni siquiera en trabajos publicados posteriormente, en la línea de la interpretación de los puntos (i) y (ii). Así, Gülich y Kotschi, años después de su primera propuesta (1995), describen esta función como un conjunto de tipologías (variación, expansión, reducción; estas últimas, a su vez, divididas en especificación y explicación, y resumen y denominación, respectivamente) según si se amplía, reduce o cambia el ámbito de denotación referencial, o si se introducen nuevos aspectos, sintetizan ideas previas, o se conceptualizan expresiones más dificiles (E. Gülich y Th. Kotschi 1987: 241-ss.; 1995: 47). Clasifican la paráfrasis únicamente como una "actividad reformuladora", sin explicitar más rasgos posibles o una relación con los conceptos de "negociación" y "completitud discursiva". Sí es clara la asociación de la reformulación no parafrástica con "alto grado de distancia", "cambio de perspectiva enunciativa", "modificación del discurso", 
e incluso su proximidad con operaciones más extremas, como la corrección o invalidación (E. Gülich y Th. Kotschi 1995: 44; C. Rossari 1990: 348, 349; C. Bach 1996: 255; M. Negroni 2009: 49).

Por tanto, existe un gran peso del "cambio de perspectiva" o de "orientación discursiva" en la noción de distancia, vinculada a una negociación y completitud discursiva que denota rasgos de una relación compleja. No obstante, este carácter discursivo no se ve reflejado en la relación de paráfrasis (F. Robles 2014: 213), a pesar de que esta tenga también una base discursiva y no pueda definirse solamente a partir de sinonimias o identidades completas entre contenidos (es decir, no se define solamente desde lo semántico o formal). La reformulación no parafrástica (la distancia), por el contrario, muestra varios grados de alejamiento semántico-contextual entre formulaciones que pueden ir del más bajo al más alto (C. Noren 1999: 49), siempre a partir de una voluntad de separarse de lo dicho teniendo en cuenta al interlocutor.

Esta información conduce a las siguientes preguntas, de carácter teórico-hipotético:

- ¿ ¿Por qué las definiciones de paráfrasis/ igualdad únicamente tienen en cuenta la no subordinación de niveles jerárquicos?

- ¿Por qué el hecho de establecer una equivalencia entre contenidos, sin que sea idéntica o completa, no puede implicar una voluntad de cambio del discurso o, al menos, de orientación formulativa con respecto a lo dicho anteriormente?

Es cierto que "igualdad" y "distancia" son diferentes (por eso existen limites entre funciones), y se ha comprobado, teórica y experimentalmente, que no son funciones iguales ni supeditadas (S. Pons 2017: 155; Autor 2019b: 173). Sin embargo, esto no quiere decir que la paráfrasis no sea una función compleja, que no exista una voluntad de negociar el discurso que la motive, y que, por tanto, sus rasgos definitorios no vayan más allá de una relación "A es igual a B en este contexto". En este punto, en el que hay dos opciones teóricas posibles, es necesario incorporar un enfoque nuevo y complementario como el que brindan los sistemas de eye-tracking desde la pragmática experimental.

\subsection{EYE-TRACKING Y PRAGMÁTICA EXPERIMENTAL}

Los métodos experimentales se han convertido en una herramienta útil para probar teorías lingüísticas y generar nuevas hipótesis teórico-prácticas (R. Gibbs 2004: 50) en varios ámbitos como la neurolingüística, la psicolingüística, la lingüística clínica, o la lingüística computacional y lenguaje artificial. Concretamente, la pragmática experimental aporta datos empíricos que apoyan, rechazan o cuestionan descripciones o intuiciones pragmático-teóricas. Su aplicación, además, contribuye a la creación de nuevos modelos para la interpretación de fenómenos pragmáticos (C. Grisot y J. Moeschler 2014: 9).

El método eye-tracking es uno de los más utilizados para estudiar fenómenos lingüísticos experimentalmente. Este método par- 
te de la llamada hipótesis ojo-mente, por la que existe una relación directa entre los movimientos oculares producidos al visualizar un contenido concreto (vídeos, imágenes, textos, párrafos, enunciados o palabras) y su procesamiento mental. En otras palabras, las reacciones de los ojos (fijaciones, pausas, movimientos hacia adelante o atrás) y, especialmente, su duración, reflejan si la información se está asimilando o no, o de qué modo (K. Rayner 1998: 374).

\subsection{HiPótesis TEÓRICO-EXPERIMENTALES}

Mediante los datos experimentales presentados en este trabajo, testaremos las siguientes hipótesis de base teórico-experimental:

a) Si la paráfrasis (y, por tanto, la relación de equivalencia) ofrece unos tiempos de lectura altos y no similares bajo diferentes condiciones, deducimos que es una función relativamente compleja.

b) Si, por el contrario, la paráfrasis ofrece unos tiempos de lectura reducidos y con mucha similitud, deduciremos que es una función de procesamiento cognitivo sencillo.

La confirmación de la hipótesis a) supone replantearse la concepción de relación de equivalencia como una actividad sencilla que no se ve afectada por el carácter discursivo de la función de la que es base; las hipótesis b) implicaría el rechazo de la anterior, un argumento más a favor de la idea de paráfrasis como función de fácil producción y asimilación. Para testar estas hipótesis, se aplicarán las siguientes medidas de lectura, basadas en la duración de los movimientos oculares.

\section{Metodología}

Los estudios clásicos de lectura y atención visual han demostrado que la visión humana refleja continuamente construcciones de representaciones mentales a partir de aquello que se observa (W. Campbell \& D. G. Green, 1965: 578). Dichas representaciones solo son posibles gracias a la precisión del ojo a través de sus componentes (retina e iris, principalmente; R.T. Addo et al., 2016: 11-12) $\mathrm{y}$ de los movimientos que realiza (horizontales y verticales, gracias a los músculos rectos y oblicuos, superiores e inferiores; K.W: Wright, 2006: 24). En conjunto, filtran la luz y crean imágenes transformadas en impulsos eléctricos que, finalmente, generan información.

Estos movimientos, en varias direcciones y con una duración determinada (fijaciones, cuando se detienen en un contenido concreto; regresiones, cuando vuelven atrás para releer o especificar un contenido; sacadas, cuando saltan hacia el siguiente contenido), se asocian a distintas fases del procesamiento lingüístico de la lectura, (morfológica, sintáctica o semántico-pragmática), y reflejan una mayor o menor dificultad de asimilación de lo observado, siempre según la hipótesis ojo-mente (K. Rayner, 1977: 443). 


\subsection{MOVIMIENTOS OCULARES Y DURACIÓN}

La mayor o menor duración de los movimientos oculares, así como el momento en el que se dan, repercuten en la comprensión y producción de un contenido lingüístico determinado (P.M. Winke, A. Godfroid y S.M. Gass, 2013: 206). Existen tres tiempos generales asociados al procesamiento cognitivo lector (K. Rayner, 2009: 4):

a) First-pass reading time (A. Duchowski, 2002: 458). Esta medida de duración tiene en cuenta la duración de todas las fijaciones acumuladas, en una palabra, parte de un texto o imagen antes de abandonarla y pasar al siguiente elemento (J. Hyöna et al., 2003: 6). Implica una primera toma de contacto con los contenidos visualizados, y el establecimiento inicial de estructuras sintáctico-semánticas, así como reconocimiento de palabras.

b) Second-pass reading time (Papadopoulou, D., \& Clahsen, H. 2003: 515). Recoge la duración de todas las fijaciones y regresiones posteriores a la primera observación del contenido. Refleja reprocesamiento y el establecimiento de supuestos pragmáticos (Baccino, 2011: 859).

c) Total reading time (M.J. Traxler \& M.J. Pickering, 1996: 460). Incluye la duración global de todos los movimientos producidos, sobre una zona en particular, durante un experimento de lectura, desde que empieza hasta que acaba. Da una visión general de una función o contenido analizado.

Su análisis es una muestra directa del procesamiento sintáctico, semántico y pragmático en la lectura: por ejemplo, el reconocimiento léxico y el asentamiento de estructuras (sintaxis, semántica) normalmente se atribuye al first-pass (T. Baccino y Y. Manunta, 2005: 204), los procesos de reanálisis (semántica, pragmática) dependen del second-pass (C. Clifton, Jr. Staub y K. Rayner, 2007: 345).

La duración permitirá determinar si la relación de equivalencia implica esfuerzos cognitivos altos o no. Si partimos de la idea de que la paráfrasis es una relación sencilla, y aplicamos la hipótesis ojo-mente, lo esperable es que ninguno de los tres tiempos de lectura de los movimientos obtenidos sea muy elevado; si, por el contrario, defendemos una visión más compleja de la función, los tiempos de lectura también presentarán ciertas particularidades.

\subsection{Diseño Del EXPERIMENTO}

\subsubsection{NÚMERO DE PARTICIPANTES}

Un experimento de lectura con eye-tracking debe realizarse con, al menos, 20 sujetos (Ó. Loureda, L. Nadal e I. Recio, 2016: 58), aunque estas cifras suelen variar ocasionalmente (algunos estudios piloto recurren a unos 10-12 participantes; otros recurren a más de 30 sujetos; S. Zufferey et al., 2018: 95). En este estudio, 
han participado 40 sujetos $^{2}$, todos con edades comprendidas entre los 18 y los 40 años, y hablantes nativos de español peninsular ${ }^{3}$.

\subsubsection{Diseño de contextos y enunciados}

El diseño de contextos y enunciados también es básico para que los resultados sean válidos. Es crucial buscar una mezcla entre cuidado en su creación y un reflejo adecuado de la función analizada, sin que parezcan textos artificiales.

Los contextos contribuyen a restringir la interpretación de los enunciados procesados y a acotar mejor y más rápido el significado de los elementos que los componen, asi como a reducir la presencia de referentes ambiguos, estructuras largas, o palabras complejas que dificulten su asimilación e incrementen tiempos de lectura innecesariamente (Wittek, Hsang Liu, Darányi, Gedeon, \& Soo Lim, 2016). Concretamente, en este estudio se han empleado dos contextos distintos para caracterizar la paráfrasis, con el fin de evitar que un participante observe el mismo material repetidamente y, por tanto, sesgar los datos:

\begin{tabular}{|c|c|}
\hline Contexto 1 & Contexto 2 \\
\hline $\begin{array}{c}\text { Estefania y Miguel son profesores de la univer- } \\
\text { sidad. Son especialistas en flora. En clase han } \\
\text { estudiado flores autóctonas y ahora quieren } \\
\text { estudiar flores exóticas. }\end{array}$ & $\begin{array}{c}\text { Lorenzo y Alejandro son fontaneros. Arreglan } \\
\text { cañerias, canalones, grifos, retretes o bañeras. }\end{array}$ \\
\hline
\end{tabular}

Tabla 1: Contextos aplicados al estudio de la paráfrasis

Estos contextos comparten la misma estructura (extensión similar, descripción sencilla, situaciones cotidianas, etc.) y contienen información directamente relacionada con los enunciados procesados. De esta manera, cuando los participantes vayan a leerlos, no partirán con dificultades de lectura iniciales que pueden resolverse.

Por otro lado, se han diseñado para el experimento cuatro enunciados parafrásticos críticos, dos con el marcador o seay otros dos sin marcador.

\begin{tabular}{|c|c|}
\hline Enunciado 1 (contexto 1) & Enunciado 2 (contexto 2) \\
\hline $\begin{array}{c}\text { Estefania y Miguel quieren una rosa rugosa; o } \\
\text { sea, japonesa. Los dos viajan a Tokio el próxi- } \\
\text { mo mes para conseguirla. }\end{array}$ & $\begin{array}{c}\text { Lorenzo y Alejandro reparan sumideros; o sea, } \\
\text { desagües. Están acostumbrados a soportar } \\
\text { malos olores. }\end{array}$ \\
\hline Enunciado 3 (contexto 1) & Enunciado 4 (contexto 2) \\
\hline $\begin{array}{c}\text { Estefania y Miguel quieren una rosa rugosa; } \\
\text { japonesa. Los dos viajan a Tokio el próximo } \\
\text { mes para conseguirla. }\end{array}$ & $\begin{array}{c}\text { Lorenzo y Alejandro reparan sumideros; des- } \\
\text { agües. Están acostumbrados a soportar malos } \\
\text { olores. }\end{array}$ \\
\hline
\end{tabular}

Tabla 2: Enunciados aplicados al estudio de la paráfrasis. Relación con cada contexto

2 Estos experimentos contaron con 160 participantes: 40 para paráfrasis, 40 para reformulación, 40 para conclusión, y 40 para corrección. Los resultados de este artículo solo se centran en uno de los varios problemas teóricos asociados a una única función, la paráfrasis; por ello solo hablamos de cuarenta individuos.

3 Se cumple así con la teoría central del límite, por la que siempre que una muestra supere los 30 sujetos podrá aceptarse experimental y estadísticamente (A. Rustom, 2012: 131). 
La presencia de cuatro enunciados para dos contextos supone que el participante, cuando realice el experimento, leerá, por ejemplo, un enunciado con marcador asociado al contexto 1 , y un enunciado sin marcador asociado al contexto 2. La distribución de contextos y enunciados, a partir del cuadrado latino, es aleatoria, por lo que los autores del experimento no pueden influir en los datos obtenidos (W.B. Wiener, 1962).

Las características de cada enunciado encajan con los requisitos estructurales propios de una paráfrasis (ver Autor, 2019b para más detalles sobre el diseño):

a) Reflejan una relación de equivalencia: un contenido más complejo, normalmente más técnico, se asocia a uno más sencillo, que se presenta como sinónimo o próximo al primero (las paráfrasis se producen, con mucha frecuencia, en discursos técnicos o de especialidad; C. Bach, 2000).

b) En los enunciados asociados al contexto 1, rosa rugosa y japonesa son los conceptos presentados como equivalentes. Dado que las rosas rugosas son típicas en Japón, se parafrasea este concepto como japonesa, lo que permite un acceso más rápido a su imagen mental. La palabra rosa no se repite para no generar costes de procesamiento extra, ya que la relación "rosa rugosa, o sea, rosa japonesa" podría interpretarse como una repetición léxica (E. Gülich y Th. Kotschi, 1983), y este no es el uso parafrástico analizado en este estudio.

c) En los enunciados del contexto 2, los conceptos sumidero y desagüe son equivalentes. Los significados de estos conceptos pueden ser más sencillos y familiares que los de rosa rugosa y japonesa; esto es intencional, ya que así los participantes podrán procesar una relación de equivalencia más compleja y otra más sencilla.

La estructura lingüística es la misma en ambos casos: un sujeto doble, basado en dos nombres propios (Lorenzo y Alejandro), un verbo en presente de indicativo (reparan), los conceptos parafraseados (sumideros; desagües), el marcador discursivo cuando se desea medir (o sea), y una pos-frase para cerrar la lectura (Están acostumbrados a soportar malos olores). Este diseño evita generar costes de procesamiento innecesarios.

\subsection{3. ÁREAS DE LECTURA MEDIDAS}

Es necesario determinar cuáles son los contenidos que leerán los participantes dentro de cada enunciado. Estas partes reciben el nombre de áreas de interés (AOI en sus siglas en inglés). En este estudio, nos centramos en las siguientes:

- $\quad$ Ä (el enunciado parafrástico completo)

- M1 (la parte parafraseada)

- M2 (el contenido que parafrasea, el equivalente)

- $\quad$ K (el marcador de reformulación; en este caso, o sea en español peninsular.

\subsubsection{TÉcnicas estadísticas (cualitativo y cuantitativo)}

Una vez obtenidos todos los datos de lectura de paráfrasis de los 40 participantes, en relación con los movimientos, tiempos (TRT, FPRT, 
SPRT), y áreas (Ä, M1, M2, K) explicados, es importante filtrarlos para su análisis. Aunque también requieren un tratamiento cualitativo, básico para poder delimitar con precisión el resultado global y cubrir objetivos de investigación específicos (J. J. Hox y H. R. Boeije, 2005: 593), sería imposible abordar todos los datos, uno a uno, sin buscar una generalización (especialmente en experimentos muy amplios). Se han aplicado modelos mixtos para el tratamiento de los datos relacionados con los tiempos de lectura (Verbeke y Molenberghs, 2000) ${ }^{4}$.

\section{Patrón cognitivo de la relación de equivalencia en paráfrasis. Datos}

Recuperamos a continuación los enunciados parafrásticos tratados en el experimento para facilitar la interpretación de los datos:

(1) Lorenzo y Alejandro reparan sumideros; o sea, desagües. Están acostumbrados a soportar malos olores.

(2) Lorenzo y Alejandro reparan sumideros; desagües. Están acostumbrados a soportar malos olores.

La siguiente imagen recoge los patrones temporales obtenidos tras el procesamiento de ambos enunciados por parte de los 40 participantes en el experimento. Cada linea representa un tiempo de lectura distinto, asociado a unas fases y características lingüísticas determinadas (3.1.), según se introduzcan o no con el marcador o sea (con marcador a la izquierda; sin marcador a la derecha). Recuérdese que M1 se refiere a "sumideros" (contenido parafraseado) y M2 a "desagües" (contenido parafraseador).

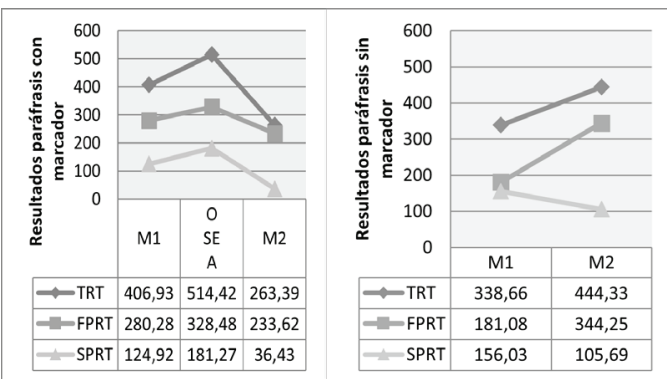

Fig. 1: Resultados procesamiento paráfrasis con y sin marcador discursivo. Patrón cognitivo. M1=sumideros; M2= desagües

A simple vista, la relación de equivalencia implica costes distintos en cada condición: mientras el marcador discursivo equilibra la acti-

4 Los modelos mixtos son una generalización de los modelos clásicos de regresión. En este caso, transforman datos temporales brutos en resultados temporales generalizables, basados en valores predictivos que se comparan para extraer similitudes y diferencias expresadas en porcentajes. Estos porcentajes contribuyen a la interpretación de los resultados en términos de efectos estadísticos altos, medios, bajos o poco relevantes Asimismo, determinan cuánto esfuerzo implica procesar la paráfrasis o hasta qué punto podemos aceptar o rechazar las hipótesis teóricas propuestas. En este modelo, los valores bajos (sobre un $5 \%$ o menores) reflejan un resultado estadístico trivial; si son más altos, la precisión de los resultados y del experimento es más fiable. 
vidad de lectura, su ausencia dispara costes cuando los participantes tienen que asimilar el contenido que parafrasea (y, por tanto, establecer dicha relación de equivalencia). Estas diferencias pueden detectarse también a través de los mapas de movimiento ocular producidos al leer ambos enunciados, conseguidos directamente en la grabación del experimento (Fig. 2): mientras el enunciado con o sea reduce notablemente las regresiones (movimientos hacia atrás para comprender lo leído) y las fijaciones (pausas sobre un contenido determinado para extraer información), se generan más esfuerzos cuando no hay un elemento procedimental que dé una instrucción sobre cómo asociar dos contenidos que deberian ser equivalentes en un contexto determinado.
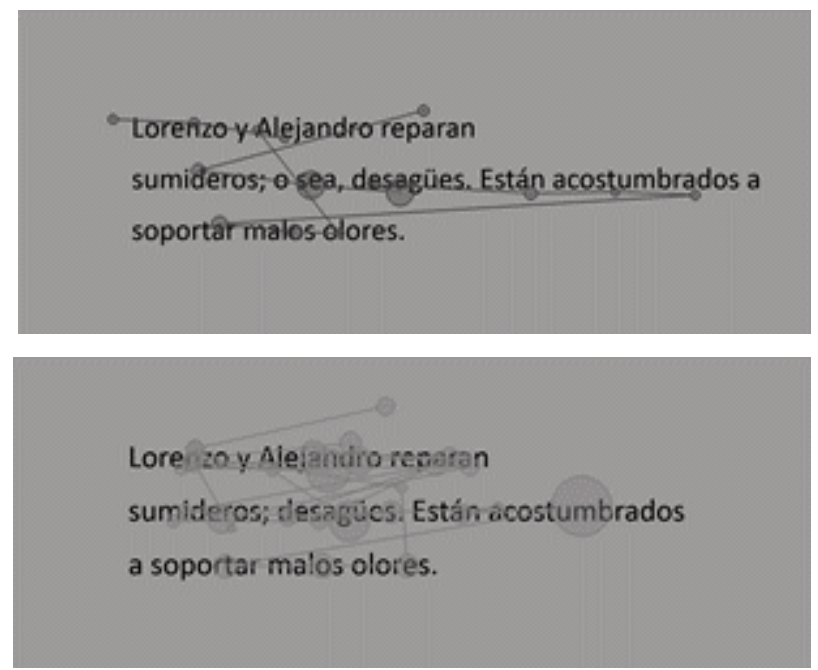

Fig. 2: Representación visual de la interpretación de una relación de equivalencia en una paráfrasis

En cualquier caso, con o sin marcador discursivo, la paráfrasis no se procesa de manera lineal. Los datos temporales asociados a estos movimientos refuerzan esta idea: dada su naturaleza semántico-discursiva, la paráfrasis implica costes de procesamiento medios-altos. El TRT (es decir, el tiempo global de procesamiento de esta función) indica que los participantes requieren alrededor de unos 300-400 ms. para asimilar la relación de equivalencia en sus diferentes componentes. Ninguno se lee tan rápidamente, ni de manera tan equilibrada, como otras funciones de carácter menos discursivo, como la conclusión (ver datos experimentales sobre la conclusión, que giran en torno a los 360 ms. para cada componente de su estructura y bajo cualquier condición; Autor, en prensa).

Asimismo, las primeras lecturas del enunciado (FPRT) concentran la mayor parte de esfuerzos cognitivos altos (como se ve en algunos valores, 280,28 ms., 233, 62 ms., 181, 08 ms., 344, 25 ms.) en comparación con su análisis pragmático, que parece más sencillo (con valores más bajos: 124, 92 ms., 36, 43 ms., 156, 03 ms., y 105, 69 ms.). En este 
sentido, puede que la relación de equivalencia plantee fases de mayor y menor dificultad: el reconocimiento léxico y la construcción de primeras presunciones comunicativas en la paráfrasis es complejo, pero, a su vez, determinante para que la asimilación pragmática se produzca fácilmente, razón por la que los costes se reducen notablemente en el SPRT.

En conjunto, los datos temporales presentan algunos picos de cifras altas y diferencias entre grupos de datos que, si bien pueden reforzar la hipótesis (a), necesitan detallarse mediante una comparación más precisa apoyada estadísticamente (porcentajes modelos mixtos). El TRT revela que el contenido que parafrasea (el M2, desagües) requiere menos costes que el contenido parafraseado (el M1, sumideros) cuando va precedido por un marcador discursivo (406, 93ms. frente a $263,69 \mathrm{~ms}$.). Sin el marcador, el esfuerzo para integrar bien desagües con sumideros es más alto $(338,66 \mathrm{~ms}$. frente a 444,33\%). En ambos casos, no se trata de una relación de paráfrasis lineal y con tiempos prácticamente idénticos, dato que apoyaría una visión de la equivalencia como relación más compleja. Esta idea se completa al comprar los grupos de datos: la palabra desagües necesita un $68,70 \%$ más de esfuerzos para su asimilación (efectos muy altos) que la misma expresada con marcador.

El FPRT muestra resultados similares. La relación es más estable cuando hay un marcador discursivo $(280,28 \mathrm{~ms}$. -sumideros- frente a 233, 62 ms. -desagües-), y se complica sin su uso (181,08 ms. -sumideros- frente a 344,25 ms. -desagües-). Generalmente, el lector tiene dificultades (más cuando no hay un marcador) para asimilar dicha relación de equivalencia desde que empieza a leerla, razón por la que no se baja de los $180 \mathrm{~ms}$. desde un primer momento (más concretamente, le cuesta comprender el tipo de relación estructural y semántica que guardan dos contenidos muy similares y que se formulan juntos, ya que tiene que desambiguar, integrar y entender bien que a pesar de predicarse una identidad entre sumidero y desagüe, este último concepto produce una reorientación discursiva para aclarar el primero, que es más técnico o complejo). De nuevo, la paráfrasis no parece ser lineal. Esta idea está apoyada por algunos datos estadísticos: p.ej., el contenido parafraseado (sumideros) cuesta un 16,65\% más que el que parafrasea (efectos estadísticos altos) cuando va precedido de un marcador discursivo, y cuando no hay instrucción procedimental, ese mismo contenido que parafrasea cuesta un 90,11\% más que el parafraseado.

Por último, el SPRT ofrece una reducción considerable de los tiempos de lectura, independientemente de que haya o no marcador (se pasa de $124,92 \mathrm{~ms}$. a 36,43 ms. en contextos con o sea, y de 156,03 ms. a 105, $69 \mathrm{~ms}$. en contextos sin o sea). Aparentemente, podría pensarse que la equivalencia no supone un esfuerzo cognitivo y que, por tanto, es una relación poco compleja. Sin embargo, esto solamente se da en la última parte de todo el proceso de lectura, una vez que los participantes han realizado las primeras interpretaciones de dicha relación y pueden tratarlo o reinterpretarlo pragmáticamente (M.V. Escandell y M. Leonetti, 2011: 93): aunque sumideros y desagües no sean completamente idénticos, el segundo es más claro que el primero porque todos accedemos más rápidamente a ese concepto que a uno más técnico, por lo 
que utilizarlo como equivalente evitará problemas comunicativos. Esta idea se sostiene con porcentajes estadísticos (diferencias de 55,43\% y $13,83 \%$; 84,41\% y 69,30\%; efectos estadísticos muy altos y altos).

\section{Conclusiones}

Según los datos obtenidos, rechazariamos la hipótesis b) y confirmaríamos la a), por la que sería necesario replantear la concepción de relación de equivalencia como una actividad sencilla, a pesar del carácter discursivo de la función a la que subyace. Los datos demuestran que la paráfrasis no es una función lineal en cuanto a su procesamiento (ver mapa de movimientos oculares), que comprende un espectro amplio de valores temporales, entre los $150 \mathrm{~ms}$. y los 500ms. (ver gráficas con los tiempos de lectura), que aumentan hasta en un $200 \%$ (en términos de efectos estadísticos) cuando un marcador discursivo no la introduce.

Cuando se define la noción de paráfrasis en relación con el concepto de equivalencia, sería necesario incorporar características como "cambio de planificación discursiva" o "completitud" y "negociación" sobre lo dicho o lo escrito, ya que, en cierto sentido, si un hablante o un escritor siente que debe aclarar un contenido para buscar la mayor comprensión por parte de su interlocutor, puede que esté marcando una ligera distancia (no en el mismo grado que el de una reformulación) con lo que ha dicho o va a decir o escribir. Y esto conlleva que la relación de equivalencia deje de estar tan marcada por una visión semántica estricta, de dos contenidos que son idénticos, para dar más importancia a la acción de predicar dicha identidad (Mortureux, 1982), que, finalmente, no es tan sencilla como pudiera parecer. En términos experimentales, si una relación de equivalencia se procesara fácilmente, los resultados habrian sido mucho más homogéneos, prácticamente iguales, con costes muy bajos y sin grandes diferencias por la presencia/ausencia de marcador discursivo.

Los estudios experimentales aplicados a la lingüística teórica no pretenden en modo alguno sustituir aquello que se ha dicho desde la teoría, sino complementar o plantear nuevas hipótesis en relación con sus objetos de estudio (Loureda et al. 2013: 75). Sin embargo, un experimento de estas características, y aplicado al problema de esta investigación, ha permitido recopilar un conjunto de datos, analizables cualitativa y cuantitativamente, que refuerzan la teoría sin recurrir únicamente a la intuición ${ }^{5}$ (A. López Serena, 2014: 693). Este es un experimento replicable bajo estas y otras condiciones (K. Popper, 1980 [1935]: 68), por lo que sería posible ampliarlo a más sujetos, añadir más grados de equivalencia para hallar más grados de complejidad en su procesamiento, o compararlo con otras funciones cercanas para refinar la relación entre teoria y datos.

5 Ver los conceptos de intuición, empirismo y empatía, analizados en A. López Serena (2014), y el debate sobre la validez de los datos frente a la intuición lingüística. 


\section{REFERENCIAS}

ANTOS, G. (1982): Grundlagen eine Theorie des Formulierens. Tubingen: Reihe Gramatische Linguistik.

BACCINO, T. \& MANUNTA, Y. (2005). Eye-Fixation-Related Potentials: Insight into Parafoveal Processing. Journal of Psychophysiology, 19(3) 204-215.

BACCINO, T. (2011): "Eye Movements and concurrent ERP's: EFRPs investigations in reading", Liversedge, S. P., Gilchrist, I. D., Everling, S (eds.), Handbook on Eye Movements, Oxford, UK: Oxford University Press, pp. 857-870.

BACH MARTORELL, C. (2000): "Mecanismos de reformulación parafrástica del catalán. Estudio de los conectores reformulativos parafrásticos és a dir (es decir), més ben dit/millor dit (mejor dicho) y dit d'una altra manera (dicho de otro modo)", Ruiz de Mendoza Ibáñez, F. J. (coord.), Panorama actual de la lingüística aplicada: conocimiento, procesamiento y uso del lenguaje, Logroño: Universidad de La Rioja, pp. 41-51. CLIFTON, C., JR., STAUB, A. \& RAYNER, K. (2007): Eye movements in reading words and sentences, van Gompel, R. P. G., Fischer, M. H., Murray, W. S. y Hill, R. L. (eds.), Eye movements: A window on mind and brain, Amsterdam: Elsevier, pp. 341-371.

DUCHOWSKI, A. (2007): Eye tracking methodology: Theory and practice. Nueva York: Springer.

ESCANDELL VIDAL, M. V. \& LEONETTI, M. (2011): "On the rigidity of procedural meaning”, Escandell. M. V., Leonetti, M. y Ahern, A. (eds.), Procedural Meaning: Problems and Perspectives,
Bingley: Emerald, pp. 81-102. GRISOT, C. \& MOESCHLER, J. (2014): How Do Empirical Methods Interact with Theoretical Pragmatics? The Conceptual and Procedural Contents of the English Simple Past and Its Translation into French, Trillo, R. (ed.), Yearbook of Corpus Linguistics and Pragmatics, 2014: New Empirical and Theoretical Paradigms, 2. Switzerland: Springer International Publishing, pp. 7-33. GÜLICH, E. \& KOTSCHI, T. H. (1983): "Les marqueurs de la réformulation paraphrastique", Connecteurs Pragmatiques et Structure du discours, Cahiers de linguistique française, 5, pp. 305-351.

GÜLICH, E. \& KOTSCHI, T. H. (1987): "Les actes de reformulation dans la consultation: La dame de Caluire", Bange, P. (ed.), L'analyse des interactions verbales. La dame de Caluire: une consultation. Berlin: Peter Lang, pp. 15-81.

HOX, J. J. \& BOEIJE, H. R. (2005): "Data collection, Primary vs. Secondary", Encyclopedia of social measurement, Amsterdam: Elsevier, Inc., pp. 593-599.

JUST, M. A. \& CARPENTER, P. A. (1980): "A theory of reading: From eye fixations to comprehension". Psychological Review, 87(4) pp. 329-354.

LÓPEZ SERENA, A. \& LOUREDA, Ó. (2013): "La reformulación discursiva entre lo oral y lo escrito: una aproximación teórica y experimental". Oralia, Análisis del discurso oral, 16, pp. 221-258.

LÓPEZ SERENA, A. (2014): "Historia de la lengua e intuición". Rilce, 30(3) pp. 691-704.

LOUREDA, Ó. ET AL. (2013): "Aproximación experimental sobre los costes de procesamiento de las partículas focales del es- 
pañol también e incluso". Cuadernos AISPI, 2, pp. 75-98.

LOUREDA, Ó., NADAL, L. \& RECIO, I. (2016a): "El significado procedimental y las partículas discursivas del español: una aproximación experimental." Revista Signos. Estudios de Lingüistica, 49, pp. 52-77.

LOUREDA, Ó, NADAL, L. \& RECIO, I. (2016b): "Partículas discursivas y cognición: 'por tanto' y la conexión argumentativa." Romanistisches Jahrbuch 67(1) Berlin: De Gruyter Mouton, pp. 240-254.

MORTUREUX, M. F. (1982): "Paraphrase et métalanguage dans le dialogue de vulgarisation", Langue française, 53, pp. 48-81.

NEGRONI, M. M. (2009): "Reformulación parafrástica y no parafrástica y ethos discursivo cognitive processes". Memory \& Cognition, 5, pp. 443-448.

RAYNER, K. (1978): "Eye movements in reading and information processing". Psychological Bulletin, 85(3) pp. 618-660.

RAYNER, K. (1998): "Eye movements in reading and information processing: 20 years of research". Psychological bulletin, 124(3) pp. 372-422.

RAYNER, K. (2009): "Eye movements and attention in reading, scene perception and visual search". Quarterly Journal of Experimental Psychology, 62(8), pp. 14571506.

ROULET, E. (1986): "Complétude interactive et mouvements discursifs". Cahiers de Linguistique Française, 7, pp. 193-210.

ROULET, E. (1987): "Completude interactive et connecteurs reformulatifs", Cahiers de linguistique française, 8, pp. 111-140.

RUSTOM, A. J. (2012): Estadistica descriptiva, probabilidad e inferencia. Una visión conceptual y aplicada. Chile: Univ. De
Chile. Disponible en http:// repositorio.uchile.cl/handle/2250/120284 (fecha de consulta: 30/11/2020).

SMABY, R. A. (1971): "Introduction", Paraphrase grammars. Formal linguistics Series, 2, 8. Dordrecht: D. Reidel Publishing Co., pp. 1-15.

TRAXLER, M. J. \& PICKERING, M. J. (1996): "Plausibility and the processing of unbounded dependencies: An eye-tracking study". Journal of Memory and Language, 35(3), pp. 454-475.

VASSILIADOU, H. (2019): "Qui trop embrasse, mal étreint: pour une conception stricte de la notion de reformulation", Landolsi, H., Svensson, M. y Noren, C. (eds.), La reformulation: à la recherche d'une frontière, Acta Upsaliensis, Studia Romanica, pp. 93-112.

VERBEKE, G. \& MOLENBERGHS, G. (2000): Linear Mixed Models for Longitudinal Data. Nueva York: Springer.

WINER, B. J. (1962): Statistical principles in experimental design. New York: McGraw Hill.

WITTEK, P., Liu, Y.-H., Darányi, S., Gedeon, T. \& Lim, I. S. (2016): "Risk and ambiguity in information seeking: Eye gaze patterns reveal contextual behavior in dealing with uncertainty". Frontiers in Psychology, 7, 1790. Disponible en: https://www.frontiersin.org/articles / 10.3389/ fpsyg.2016.01790/full (Fecha de consulta: 30/11/2020).

ZUFFEREY, S., MAK, W., VERBRUGGE, S. \& SANDERS, T. (2018): "Usage and processing of the French causal connectives 'car' and "parce que." Journal of French Language Studies, 8(1), pp. 85-112. 